\title{
Medial Frontal Cortex Mediates Perceptual Attentional Set Shifting in the Rat
}

\author{
Jennifer M. Birrell and Verity J. Brown \\ School of Psychology, University of St. Andrews, St. Andrews KY16 9JU, Scotland, United Kingdom
}

If rodents do not display the behavioral complexity that is subserved in primates by prefrontal cortex, then evolution of prefrontal cortex in the rat should be doubted. Primate prefrontal cortex has been shown to mediate shifts in attention between perceptual dimensions of complex stimuli. This study examined the possibility that medial frontal cortex of the rat is involved in the shifting of perceptual attentional set. We trained rats to perform an attentional set-shifting task that is formally the same as a task used in monkeys and humans. Rats were trained to dig in bowls for a food reward. The bowls were presented in pairs, only one of which was baited. The rat had to select the bowl in which to dig by its odor, the medium that filled the bowl, or the texture that covered its surface. In a single

Whether there is a homolog of primate prefrontal cortex on the medial wall of frontal cortex of the rodent brain is an issue of continuing controversy (Kolb, 1990; Preuss, 1995). There are extensive projections from the mediodorsal nucleus of the thalamus to medial frontal cortex and these projections are generally assumed to define rodent prefrontal cortex. Nevertheless, in primate, areas other than prefrontal cortex also receive projections from mediodorsal nucleus (for example, anterior cingulate cortex). Thus, receipt of mediodorsal thalamic projections cannot be accepted as evidence that an area of rodent cortex is homologous to primate prefrontal cortex as opposed to, for example, cingulate cortex (Preuss, 1995). An alternative approach to the issue is to consider the function, rather than anatomy, of frontal areas. The evolution of prefrontal cortex in the primate is, presumably, to support a degree of behavioral complexity that might not be found in the rat. However, if the rodent does demonstrate behavior dependent on functions associated with primate prefrontal cortex, the neural substrate of such behavior could be regarded as at least analogous, if not homologous, to primate prefrontal cortex.

In primates, prefrontal cortex mediates shifts in attention between perceptual features of complex stimuli (Owen et al., 1991; Dias et al., 1996a,b, 1997). When attending to a perceptual feature (e.g., color) of a stimulus, learning to discriminate novel complex stimuli is more rapid when the discrimination rule is

Received Jan. 24, 2000; revised March 13, 2000; accepted March 20, 2000.

This work was supported by The Wellcome Trust (Project Grant 051945/Z/97/Z). J.B. received a Biotechnology and Biological Sciences Research Council (UK) studentship. We thank the animal care staff of the School of Psychology Animal House; Andrew Blackwell, Dr. Janice Phillips, and William Robb for assistance during the development of the behavioral task; and Dr. Angela C. Roberts for helpful discussion.

Correspondence should be addressed to Dr. Verity J. Brown, School of Psychology, University of St. Andrews, St. Andrews KY16 9JU, Scotland, UK. E-mail: vjb@st-and.ac.uk.

Copyright (C) 2000 Society for Neuroscience $\quad 0270-6474 / 00 / 204320-05 \$ 15.00 / 0$ session, rats performed a series of discriminations, including reversals, an intradimensional shift, and an extradimensional shift.

Bilateral lesions by injection of ibotenic acid in medial frontal cortex resulted in impairment in neither initial acquisition nor reversal learning. We report here the same selective impairment in shifting of attentional set in the rat as seen in primates with lesions of prefrontal cortex. We conclude that medial frontal cortex of the rat has functional similarity to primate lateral prefrontal cortex.

Key words: attention; set shifting; prefrontal cortex; Cg3; prelimbic cortex; rat

based on the same perceptual dimension [an intra-dimensional (ID) shift]. By contrast, if the new discrimination requires that attention is directed to a different perceptual dimension (e.g., the form of the stimulus) and the previously attended feature must be disregarded [an extra-dimensional (ED) shift], the new discrimination is acquired less rapidly. Lesions of primate lateral prefrontal cortex result in normal acquisition of attentional set but impairment on the ED shift (Dias et al., 1996a,b, 1997).

Rats with lesions of medial frontal cortex are impaired in shifting between response rules (Joel et al., 1997; Ragozzino et al., 1999a,b), which may reflect an attentional set-shifting deficit. Attentional deficits have also been proposed to account for impairments in reversal learning of difficult to discriminate stimuli (Bussey et al., 1997) and delayed response tasks (Delatour and Gisquet-Verrier, 1999). However, a compelling demonstration of a deficit of attentional set-shifting would be to show that medial frontal cortex lesions result in specific deficits in shifting of selective attention for perceptual features of stimuli. This would require a task that is formally the same as the task used in humans and monkeys: a two-choice discrimination using complex stimuli that differ along several perceptual dimensions. Here we report the effects of lesions of rodent medial frontal cortex on performance of such a task.

\section{MATERIALS AND METHODS}

Animals. Twenty-four Lister hooded rats (Charles River, Margate, Kent, UK) were housed individually in $25 \times 45 \times 15 \mathrm{~cm}$ plastic cages. Testing was conducted in the light phase of a $12 \mathrm{hr}$ light/dark cycle (lights on at 7 A.M.). The rats were maintained on a restricted diet (15-20 gm of food per day) with water freely available in the home cage. We adhered to the guidelines laid out in the Principles of Laboratory Animal Care (National Institutes of Health, Publication No. 86-23, revised 1985) and the requirements of the United Kingdom Animals (Scientific Procedures) Act 1986.

Apparatus. Rats can be trained to dig in small bowls filled with sawdust to retrieve food reward (Wood et al., 1999). The digging bowls used here 
Table 1. Order of discriminations, which was the same for all rats

\begin{tabular}{|c|c|c|c|c|}
\hline \multirow[b]{2}{*}{ Discriminations } & \multicolumn{2}{|c|}{ Dimensions } & \multicolumn{2}{|c|}{ Exemplar combinations } \\
\hline & Relevant & Irrelevant & + & - \\
\hline Simple (SD) & Odor & & 01 & $\mathrm{O} 2$ \\
\hline \multirow[t]{2}{*}{ Compound (CD) } & Odor & Medium & O1/M1 & $\mathrm{O} 2 / \mathrm{M} 2$ \\
\hline & & & O1/M2 & $\mathrm{O} 2 / \mathrm{M} 1$ \\
\hline \multirow[t]{2}{*}{ Reversal (Rev1) } & Odor & Medium & O2/M1 & $\mathrm{O} 1 / \mathrm{M} 2$ \\
\hline & & & $\mathrm{O} 2 / \mathrm{M} 2$ & $\mathrm{O} 1 / \mathrm{M} 2$ \\
\hline \multirow[t]{2}{*}{ Intradimensional shift (ID) } & Odor & Medium & $\mathbf{0 3} / \mathrm{M} 3$ & $\mathrm{O} 4 / \mathrm{M} 4$ \\
\hline & & & O3/M4 & $\mathrm{O} 4 / \mathrm{M} 3$ \\
\hline \multirow[t]{2}{*}{ Reversal (Rev2) } & Odor & Medium & O4/M3 & $\mathrm{O} 3 / \mathrm{M} 4$ \\
\hline & & & O4/M4 & $\mathrm{O} 3 / \mathrm{M} 3$ \\
\hline \multirow[t]{2}{*}{ Extradimensional shift (ED) } & Medium & Odor & M5/O5 & M6/O6 \\
\hline & & & M5/O6 & $\mathrm{M} 6 / \mathrm{O} 5$ \\
\hline \multirow[t]{2}{*}{ Reversal (Rev3) } & Medium & Odor & M6/O5 & M5/O6 \\
\hline & & & M6/O6 & $\mathrm{M} 5 / \mathrm{O} 5$ \\
\hline
\end{tabular}

Examples of combinations into stimulus pairs are shown for a rat shifting from odor to digging medium. On every trial, the pair of stimuli differed on both the relevant and irrelevant dimensions. The correct exemplar is shown in bold, paired with either exemplar from the irrelevant dimension. The combination of exemplars into positive $(+)$ and negative $(-)$ stimuli and their left-right position of presentation in the cage was a pseudorandom series determined in advance.

were ceramic pots, with an internal diameter of $7 \mathrm{~cm}$ and a depth of $4 \mathrm{~cm}$. The bait was one-half of a Honey Nut Loop (Kellogg, Manchester, UK). The outer surface and rim of the bowl were covered with a texture, and the bowls were filled with a digging medium, which could be scented. Thus, the bowls could be varied by their odor, the texture of the outer surface and rim of the bowl, or the digging medium in which the food bait was hidden.

The test apparatus was an adapted plastic cage $(40 \times 70 \times 18 \mathrm{~cm})$ with Plexiglas panels used to divide one-third of the length of the cage into two sections. The digging bowls were placed in these sections, with a central divider between them. A removable divider separated the rat from the two sections in which the bowls were placed. The rat could be given access to the bowls by lifting this divider. The purpose of the dividers was to enable the experimenter to block access to the bowls, particularly after an error; without the dividers, a rat could move quickly between the bowls and retrieve the bait before the experimenter had time to remove it.

Habituation. On the day before testing, rats were given access to two sawdust-filled bowls in the testing cage for $60 \mathrm{~min}$. Both of the bowls were rebaited every $5 \mathrm{~min}$. When the rat was reliably digging to retrieve the rewards, it was trained on a series of three simple discriminations (SDs) (texture of rubber vs masking tape; odor of blackcurrant vs vanilla; digging medium of styrofoam vs shredded paper) to a criterion of six consecutive correct trials. All rats were trained on the same discriminations, in the same order. The exemplars were not used again.

Testing paradigm. A trial was initiated by raising the divider to give the rat access to the two digging bowls, only one of which was baited. The first four trials were discovery trials: the rat was permitted to dig in both of the bowls, but only one was baited. An error was recorded if the rat dug first in the unbaited bowl. On subsequent trials, if the rat started to dig in the unbaited bowl, an error was recorded, and the trial was terminated. Testing continued until the rat reached a criterion level of performance of six consecutive correct trials.

In a single test session, rats performed a series of discriminations that paralleled those used in the equivalent task for primates (Table 1). In the
$\mathrm{SD}$, the bowls differed along one of three dimensions (e.g., odor or texture or digging medium). For the compound discrimination (CD), a second dimension was introduced, but the correct and incorrect exemplars remained constant. For the reversals (Rev1, Rev2, and Rev3), the exemplars and the relevant dimension were unchanged: the rat had to learn that the previously correct stimulus was now incorrect. For both the ID and ED shifts, there were new exemplars of both the relevant and irrelevant dimensions (a total change design); in the latter case, the previously relevant dimension was now the irrelevant dimension. The order of the discriminations was always the same, but the dimensions and the pairs of exemplars were equally represented within groups and counterbalanced between groups as far as possible.

There were six possible patterns of shift (odor to texture or medium, medium to odor or texture, texture to medium or odor), so each pattern of shift was used twice in each group. The combinations of exemplars were too numerous to permit full counterbalancing; therefore, the following procedure was used. To reduce the degrees of freedom, exemplars were always used in pairs; for example, if cumin were the positive stimulus, the negative stimulus was always cinnamon (Table 2). This also meant that exemplars of texture and digging medium could be matched for their odors and visual appearance; thus, grades of tea were used as a pair of exemplars of digging medium, and reverse sides of velvet cloth were used as a pair of exemplars of texture. Although pairs were still too numerous to test all combinations, no two rats within groups received the same combinations, but the lesion and control groups were matched. The combining of exemplars into stimuli and the side of stimulus presentation were determined by an a priori pseudorandom list.

Surgery. Anesthesia was induced with an intraperitoneal injection of pentobarbitone sodium $(1.0 \mathrm{ml} / \mathrm{kg}, 65 \mathrm{mg} / \mathrm{ml})$. The rats were then placed in a stereotaxic frame with atraumatic ear bars (Kopf, Tujunga, CA), with the nose bar set at $+5 \mathrm{~mm}$. The ibotenic acid was infused manually using a $1 \mu \mathrm{l}$ syringe, at a rate of $0.1 \mu \mathrm{l}$ every $3 \mathrm{~min}$. Twelve rats each received four injections of $0.2 \mu \mathrm{l}$ of $0.06 \mathrm{M}$ ibotenic acid (Tocris Cookson, Avonmouth, UK), bilaterally at the following coordinates relative to Bregma: anteroposterior (AP) +3.5; mediolateral (ML) \pm 0.6 ; dorsoven-

Table 2. Exemplars used

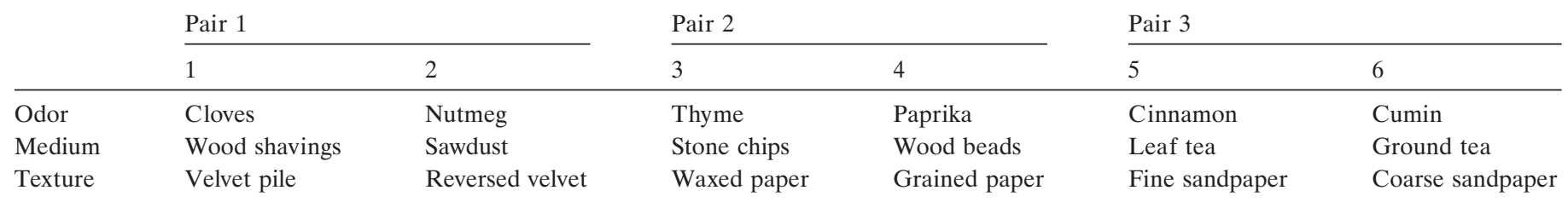

The exemplars within a dimension were presented in pairs and varied such that no two animals within a group received the same discriminations, but the shifts performed by each lesioned rat were matched by those of a control rat. 


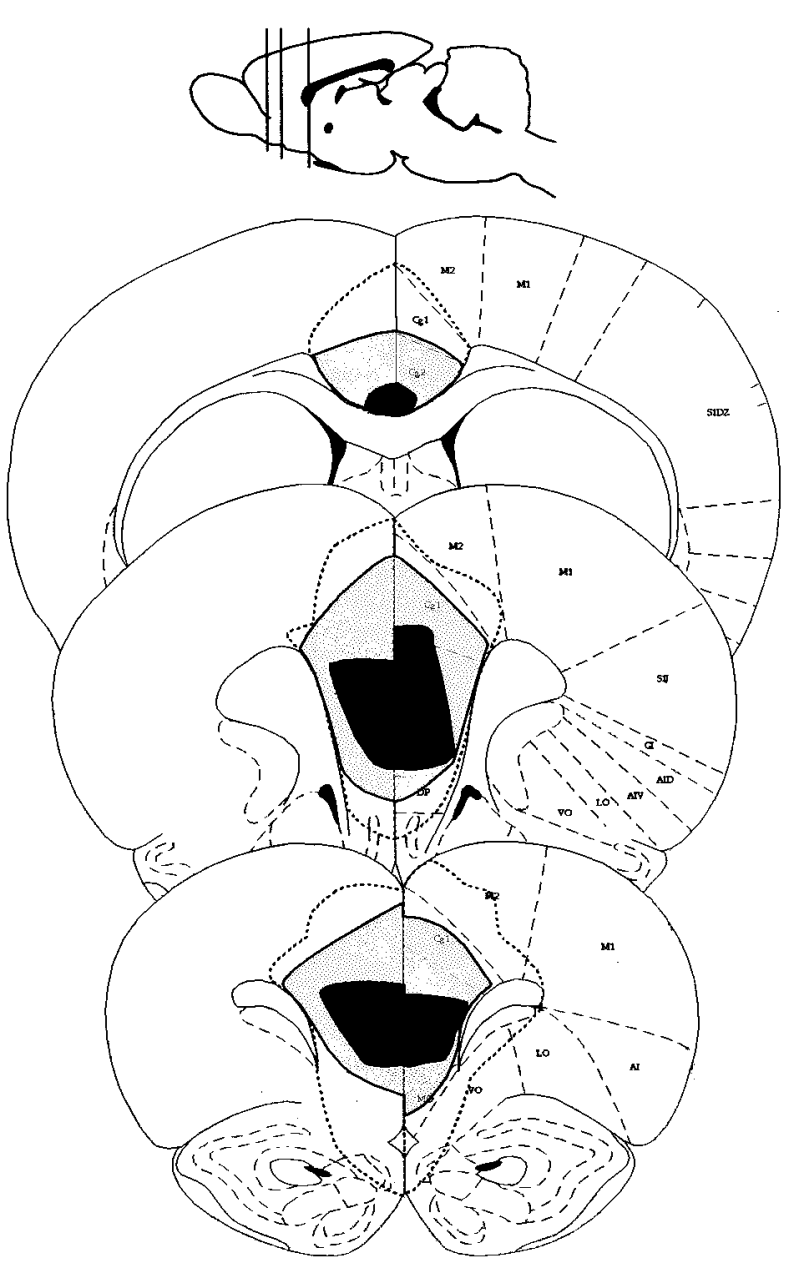

Figure 1. A series of coronal sections (adapted from Paxinos and Watson, 1997) at 3.7, 2.7, and $1.0 \mathrm{~mm}$ anterior to bregma. The extent of the area of damage common to all lesioned rats is shown in black, whereas the area of damage common to $50 \%$ of rats is shown shaded. The maximum extent of any damage is shown as a dotted line.

tral (DV) -5.2 ; and $\mathrm{AP}+2.5 ; \mathrm{ML} \pm 0.6 ; \mathrm{DV}-5.0$. Control rats received inf usions of vehicle at the same coordinates. The syringe was left in place for $4 \mathrm{~min}$ before being withdrawn slowly. Testing was conducted on day 5 after surgery.

Histology. The rats were killed by intraperitoneal administration of Euthanol $(1.0 \mathrm{ml} / \mathrm{kg}$, pentobarbitone sodium, $200 \mathrm{mg} / \mathrm{ml})$. The rats were perfused transcardially with phosphate buffer for $2 \mathrm{~min}$ at a rate of 10 $\mathrm{ml} / \mathrm{min}$, followed by a $4 \%$ paraformaldehyde in phosphate buffer for 20 $\min$ at the same rate. The brains were removed and placed into a $20 \%$ sucrose $/ 4 \%$ paraformaldehyde phosphate buffer solution until processed. Using a freezing microtome, $50 \mu \mathrm{m}$ coronal sections were saved every $400 \mu \mathrm{m}$ for staining with cresyl violet.

Data analysis. Trials to criterion and errors to criterion were recorded for each rat for each discrimination; however, because the two measures are correlated and analysis of either measure produced the same results, only trials to criterion are reported. Repeated-measures ANOVA was used, with three factors, one within-subjects (shift: SD, CD, Rev1, ID, Rev2, ED, Rev3) and two between-subjects (group: lesion and control; dimension change: odor to medium, odor to texture, medium to odor, medium to texture, texture to odor, and texture to medium). Planned comparisons were performed to test the source of significant interactions. Separate analyses were performed on the discriminations before the ED shift (to test the effect of initial dimension on acquisition) and the reversal discriminations (to compare initial acquisition and the three reversals).

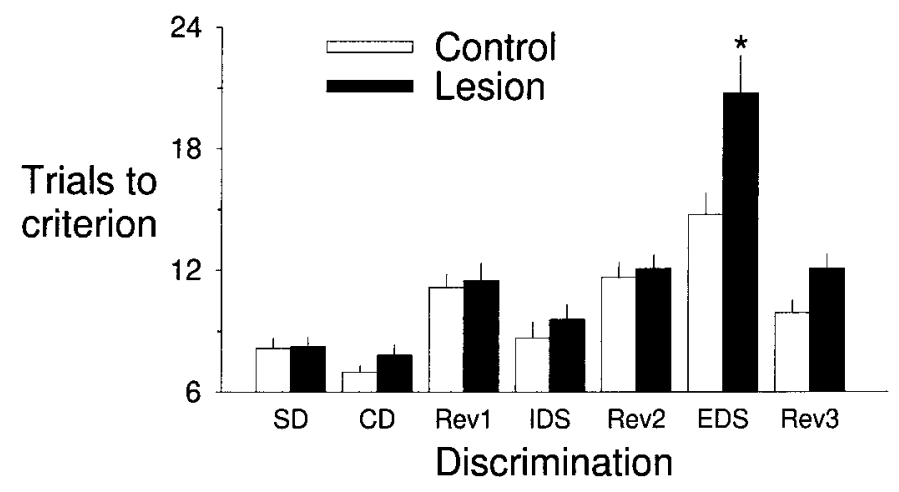

Figure 2. Bar graph showing number of trials to a criterion (six consecutive correct trials) for each discrimination. ID shifts were learned more rapidly than ED shifts. The lesions resulted in a selective impairment in the ED shift; * $p<0.05$ compared with control.

\section{RESULTS}

\section{Histology}

Twelve rats sustained bilateral damage of medial frontal cortex. Figure 1 is a series of coronal sections (adapted from Paxinos and Watson, 1997) showing the area of damage that was common to all rats as well as the areas common to $50 \%$ of rats. A boundary of damage is shown, representing not the largest lesion but the maximum extent of any lesion. Using the sections and nomenclature of Paxinos and Watson (1997), the lesions were centered on prelimbic cortex (PrL), but in all cases included damage to infralimbic cortex (IL). In half of the rats, the lesions extended dorsally to include anterior portions of cingulate cortex, $\mathrm{Cg} 1$ and $\mathrm{Cg} 2$. In two rats, there was minor damage to anterior M2.

\section{Discrimination learning}

Figure 2 shows the trials to criterion for each of the discriminations. On average, rats learned the simple discrimination to the criterion of six correct consecutive trials, in just eight trials. Analysis of the stages before the ED shift confirmed that performance did not significantly differ within the three perceptual dimensions (main effect of initial dimension, $F_{(2,18)}=1.12$, NS). There was no effect of the lesion on these initial stages (main effect of group, $F_{(1,18)}=0.95$, NS; group by stage interaction, $\left.F_{(1,18)}=1.67, \mathrm{NS}\right)$.

\section{Reversal learning}

After the CD, ID, and ED stages, the correct and incorrect exemplars were reversed. All rats required more trials to learn the reversals than they required for either initial acquisition (SD and CD stages) or the ID shift, also a novel discrimination (main effect of reversal, $\left.F_{(1,22)}=92.5, p<0.05\right)$. It was not possible to divide performance into epochs corresponding to a period of perseverative responding and a period of learning (Hunt and Aggleton, 1998), because learning was so rapid. There was a mean of 5.4 trials preceding the six criterion trials and a mean of 3.9 errors to criterion. Thus, most of the trials were perseverative. There was no effect of the lesion on reversal learning (interaction of group and reversal: $\left.F_{(1,22)}=0.3, \mathrm{NS}\right)$.

\section{ID versus ED shifts}

Learning a novel discrimination was faster when the discrimination was based on the previously relevant perceptual dimension (an ID shift) compared with an ED shift, when attention had to be shifted to the previously irrelevant dimension. All rats per- 
formed ID shifts more rapidly than ED shifts (planned contrast, ID vs ED, after main effect of shift, $\left.F_{(1,12)}=89.49, p<0.05\right)$, demonstrating that the rats formed a perceptual attentional set. Each dimension change (i.e., odor to medium or texture, medium to odor or texture, texture to odor or medium) was equivalent with respect to the ease of shifting attentional set (main effect of dimension change, $F_{(5,12)}=1.31$, NS).

Medial frontal cortex lesions resulted in a selective impairment in the ED shift, with lesioned rats taking twice as many trials as controls to learn the new discrimination (lesion by shift interaction, $F_{(6,72)}=3.48, p<0.05$; planned contrasts indicated the lesion effect was restricted to the ED shift, $\left.F_{(1,12)}=7.2, p<0.05\right)$.

\section{DISCUSSION}

Lesions of rat medial frontal cortex, centered on PrL and IL, resulted in a selective impairment of an ED shift, with no impairment in acquisition or reversal learning. These results indicate that medial frontal cortex contributes to extra-dimensional attentional set-shifting in the rat. Although half of the rats also had damage extending to $\mathrm{Cg} 1-2$, the presence of damage more dorsally was not associated with greater deficits.

Previous authors have demonstrated that rule-shifting is impaired in rats with lesions of medial frontal cortex (Joel et al., 1997; Ragozzino et al., 1999a,b). Although impaired shifting of attentional set might result in a deficit in shifting response rule, it is not necessarily the case that a deficit in shifting response rule must be caused by an impairment of attentional set. This study extends previous work in showing that lesions of medial frontal cortex result in deficits in the shifting of perceptual set.

\section{Validation of the test}

An attentional set is formed when complex stimuli must be classified or discriminated, to enhance the efficiency of processing of currently relevant features of a stimulus and enable irrelevant differences between stimuli to be ignored. The class of attended or ignored features is referred to as a stimulus dimension. This may be some perceptual attribute of the stimulus (such as the color or the shape of a visual object), but any other attribute by which stimuli may be classified and discriminated is also a stimulus dimension (for example, a semantic attribute, such as words and non-words). The problem of demonstrating perceptual attentional set in animals is designing an appropriate task. It is a challenge to devise stimuli with species-appropriate dimensions and a sufficient number of exemplars of each dimension to allow within-subject testing of all shifts, with novel stimuli at every shift (a total change design). Without a total change design, the interpretation of the superiority of reversal learning over ED shifts, as demonstrated by Mackintosh (1965), is ambiguous (Slamecka, 1968). Thus, to test ID and ED shifts, these requirements necessitate a minimum of two dimensions with six exemplars of each dimension.

The dimensions must be species-appropriate, to achieve rapid learning that is preferably equivalent for each dimension. Shepp and Eimas (1964) found that rats took more than 1 week of 25 trials per day to learn to discriminate visual stimuli by pattern or shape. Furthermore, attending to the shape of a stimulus retarded a subsequent shift of attention to its pattern more than was the case for the converse shift. The particular dimensions we used (odor, texture, or digging medium) were without differential effect on performance: at the CD stage of testing, none of the newly introduced irrelevant dimensions exerted greater distraction and neither was distractibility greater for any particular relevant dimension. Furthermore, each type of ED shift (odor to or from texture, medium to or from texture, and odor to or from medium) was learned with an equivalent number of trials. This finding has implications for the use of odor stimuli in memory tasks. It might be argued that odor stimuli are unsatisfactory because the rat may "carry" the odor on its fur to a subsequent trial, so aiding discrimination. However, the fact that the odor discrimination was not learned more rapidly than texture or digging medium (neither of which could easily be transferred to subsequent trials) suggests that rats do not use this strategy, and thus odor discriminations are suitable for use in memory tasks.

The task as used in humans and monkeys involves two dimensions that are different visual features of the stimulus. This contrasts with our task, which uses somatosensory and olfactory features of the stimulus. In the marmoset, the two dimensions used are filled shapes versus configurations of lines superimposed on the shapes. Obviously, these are both shape discriminations. What makes them separate dimensions is that they are stimulus features that are not related: shapes A and B can be paired with either line 1 or line 2, and at any time only one aspect of the pair (shape or line) is relevant. We considered the possibility that the rat might discriminate the texture or the digging medium by using visual or olfactory, rather than somatosensory, cues. We tried to minimize this possibility by choosing pairs of exemplars of texture with the same color and odor (reverse sides of dark velvet cloth, for example). However, such a procedure is not actually necessary. If the rat is able to discriminate one of two textured bowls, and ignore another irrelevant feature that might be associated with either, it is not relevant to the procedure which sensory system the rat is using. Furthermore, if this ability to discriminate the bowls according to one feature retards the ability to learn to discriminate the two bowls according to another feature, we can conclude that an attentional set is formed. The contents of attentional set transcend sensation and sensory modality.

\section{Medial frontal lesions and reversal learning}

Previous work has reported deficits in reversal learning after lesions of medial frontal cortex (for review, see Kolb, 1990), but Bussey et al. (1997) showed that reversal learning was only impaired when the stimuli were difficult to discriminate. Bussey et al. (1997) suggest that this reversal deficit might be caused by impairment in the ability to attend to relevant stimulus features. Here, we replicate their result that medial frontal lesions result in no impairment in reversal learning of easily discriminable stimuli, and we provide evidence to suggest that their deficit is indeed likely to be of one selective attention.

Reversals are a special case of an intradimensional shift. Like the ID shift, reversal learning does not require attention to be reoriented, because the attended dimension remains the same. However, unlike the ID shift in which all the exemplars are novel, in the reversal, the exemplars remain the same: the previously correct exemplar is now incorrect, and the rat must respond to the previously incorrect exemplar. Any tendency to persevere in responding to a previously correct stimulus would impair reversal learning. All rats required more trials to learn the reversals than they required for either initial acquisition (SD and CD stages) or an ID shift in which all the stimuli were novel. However, there was no effect of the lesion of medial frontal cortex on reversal learning. This indicates that the ED shift deficit is not caused by a problem with perseverative responding in a general sense, because the rats are as able as controls to abandon a response to 
a previously reinforced stimulus. Rather, perseveration is restricted to the shifting of attention.

\section{Medial frontal cortex lesions and set-shifting}

All rats performed ID shifts more rapidly than ED shifts, demonstrating that the rats formed a perceptual attentional set. The rats with medial frontal cortex lesions showed retardation in the shifting of attentional set, doubling the number of errors at this shift. It should be noted that although the discrimination was learned more slowly, it was not a more difficult discrimination: only previous experience of attending to the now irrelevant dimension distinguished the new learning at the ED shift from that at the ID stage. Therefore, the lesion effect cannot be attributed to an effect of discrimination difficulty, other than that resulting from the necessity to shift attentional set.

The result we report here is directly comparable, in nature as well as magnitude, to the set-shifting deficit reported in marmosets after lesions of lateral frontal cortex (Dias et al., 1996a,b, 1997). Consequently, this task provides the opportunity to study the neuropsychological basis of attentional set-shifting in rats, which has many practical advantages over primates. Because our task also includes a third dimension, it will be possible in the future to examine the nature of the deficit in terms of learned irrelevance and perseveration (Owen et al., 1993; GauntlettGilbert et al., 1999), which is not possible in a task with just two dimensions.

Using this measure of perceptual attentional set-shifting in the rat, the work reported here provides compelling evidence to support what has already been suggested in the literature. Namely, that rat medial frontal cortex is not merely concerned with spatial tasks or working memory function, but rather, that the deficits reflect impairment of a supervisory attentional system (Bussey et al., 1997). This suggests that rat medial frontal cortex contains a homolog of primate prefrontal cortex. Furthermore, this study has developed a suitable task for use in the rat, so that investigations into the neural basis of perceptual attentional set need no longer be restricted to primates.

\section{REFERENCES}

Bussey TJ, Muir JL, Everitt BJ, Robbins TW (1997) Triple dissociation of anterior cingulate, posterior cingulate, and medial frontal cortices on visual discrimination tasks using a touchscreen testing procedure for the rat. Behav Neurosci 111:920-936.

Delatour B, Gisquet-Verrier P (1999) Lesions of the prelimbic- infralimbic cortices in rats do not disrupt response selection processes but induce delay-dependent deficits: evidence for a role in working memory? Behav Neurosci 113:941-955.

Dias R, Robbins TW, Roberts AC (1996a) Dissociation in prefrontal cortex of affective and attentional shifts. Nature 380:69-72.

Dias R, Robbins TW, Roberts AC (1996b) Primate analogue of the Wisconsin Card Sorting Test: effects of excitotoxic lesions of the prefrontal cortex in the marmoset. Behav Neurosci 110:872-886.

Dias R, Robbins TW, Roberts AC (1997) Dissociable forms of inhibitory control within prefrontal cortex with an analog of the Wisconsin Card Sort Test: restriction to novel situations and independence from "online” processing. J Neurosci 17:9285-9297.

Gauntlett-Gilbert J, Roberts RC, Brown VJ (1999) Mechanisms underlying attentional set-shifting in Parkinson's disease. Neuropsychologia 37:605-616.

Hunt PR, Aggleton JP (1998) Neurotoxic lesions of the dorsomedial thalamus impair the acquisition but not the performance of delayed matching to place by rats: a deficit in shifting response rules. J Neurosci 18:10045-10052.

Joel D, Weiner I, Feldon J (1997) Electrolytic lesions of the medial prefrontal cortex in rats disrupt performance on an analog of the Wisconsin Card Sorting Test, but do not disrupt latent inhibition: implications for animal models of schizophrenia. Behav Brain Res 85:187-201.

Kolb B (1990) Prefrontal cortex. In: The cerebral cortex of the rat (Kolb B, Tees RC, eds), pp 437-458. Boston: MIT.

Mackintosh NJ (1965) Selective attention in animal discrimination learning. Psychol Bull 64:423-438.

Owen AM, Roberts AC, Polkey CE, Sahakian BJ, Robbins TW (1991) Extra-dimensional versus intra-dimensional set shifting performance following frontal-lobe excisions, temporal-lobe excisions or amygdalohippocampectomy in man. Neuropsychologia 29:993-1006.

Owen AM, Roberts AC, Hodges JR, Summers BA, Polkey CE, Robbins TW (1993) Contrasting mechanisms of impaired attentional set shifting in patients with frontal-lobe damage or Parkinson's disease. Brain 116:1159-1175.

Paxinos G, Watson C (1997) The rat brain in stereotaxic coordinates, Ed 3. Sydney: Academic.

Preuss TM (1995) Do rats have prefrontal cortex? The Rose-WoolseyAkert Program reconsidered. J Cognit Neurosci 7:1-24.

Ragozzino ME, Detrick S, Kesner RP (1999a) Involvement of the prelimbic-infralimbic areas of the rodent prefrontal cortex in behavioral flexibility for place and response learning. J Neurosci 19:4585-4594.

Ragozzino ME, Wilcox C, Raso M, Kesner RP (1999b) Involvement of rodent prefrontal cortex subregions in strategy switching. Behav Neurosci 113:32-41.

Shepp BE, Eimas PD (1964) Intradimensional and extradimensional shifts in the rat. J Comp Physiol Psychol 57:357-361.

Slamecka NJ (1968) A methodological analysis of shift paradigms in human discrimination learning. Psychol Bull 69:423-438.

Wood ER, Dudchenko PA, Eichenbaum H (1999) The global record of memory in hippocampal neuronal activity. Nature 397:613-616. 